\title{
Bioanalysis
}

\section{Technologies and strategies for bioanalysis of biopharmaceuticals}

\author{
"...the articles in this special focus issue of Bioanalysis of \\ Biopharmaceuticals highlight some recent advances in technologies \\ and strategies to address both bioanalytical capability and capacity \\ gaps in support of emerging complex biopharmaceuticals and \\ biosimilars."
}

First draft submitted: 14 July 2017; Accepted for publication: 4 August 2017; Published online: TBC

Keywords: analytical analysis $\bullet$ bioanalysis $\bullet$ biopharmaceuticals $\bullet$ biosimilar - biotherapeutics $\bullet$ gene therapy $\bullet$ immunogenicity $\bullet$ ligand-binding assay

- mass spectrometry $\bullet$ tissue analysis

Biopharmaceuticals are gaining more interest as new therapeutic agents due to their high selectivity for respective targets and potential to treat life-threatening and orphan diseases that are retained from traditional small molecule therapy [1]. Major classes of biopharmaceuticals include hormones, monoclonal antibodies (mAbs), growth factors, vaccines, cytokines, blood factors and others including gene and cell therapy biological products [1] Of them, protein therapeutics now represent the second largest biopharmaceutical product category after vaccines [2]. In particular, mAbs constitute the fastest growing sector within the therapeutic protein industry, resulting in occupying about half of the biopharmaceutical market [3]. The success of $\mathrm{mAbs}$ in the pharmaceutical market has triggered the development of various antibody-based therapeutics such as multispecific mAbs, antibody-drug conjugates, and Fc fusion proteins with an aim of increasing target-binding affinity and selectivity, extending half-life, and reducing immunogenicity and toxicity [1,4-5]. In addition, the field has turned to so-called 'alternative protein scaffolds', which are smaller and more modular than their antibody counterparts, to address the limitation of antibodies [5]. In parallel, the patents related top-selling biopharmaceuticals have reached their expi- ration and more will expire within the next few years [6], resulting in explosive creation of opportunities for biosimilar manufacturers to market their own generic versions of biopharmaceuticals [7,8]. Furthermore, DNAand RNA-based therapeutics have also grown rapidly alongside protein therapeutics $[9,10]$.

As technology progresses, biopharmaceuticals continue to increase in complexity, placing expanding demand for bioanalytical capability to assess drugZ pharmacology, safety and immunogenicity. In addition, a growing number of biosimilars require approaches that are scientifically sound, harmonized across the industry and accepted by the regulatory agencies to assess the compatibility with their respective originators $[11,12]$. Furthermore, the increase in the number of biopharmaceuticals calls for strategies to balance quality, speed and cost. The articles in this Special Focus Issue on 'Bioanalysis of Biopharmaceuticals' highlight some recent advances in technologies and strategies to address both bioanalytical capability and capacity gaps in support of emerging complex biopharmaceuticals and biosimilars. The topics include quantification or characterization of a variety of complex biopharmaceuticals from oligonucleotide-based drugs to protein therapeutics with post-translation modification (PTM) in biological fluids or tissues, introducing different bioanalytical
Yan J Zhang*,1 \& Hyun Joo $\mathrm{An} * *, 2,3$

'Bioanalytical Sciences, Analytical \& Bioanalytical Operations, Bristol-Myers Squibb, Princeton, NJ 08543, USA ${ }^{2}$ Graduate School of Analytical Science \& Technology, Chungnam National University, Daejeon, Korea

${ }^{3}$ Asia Glycomics Reference Site, Daejeon, Korea

*Author for correspondence: yan.j.zhang@bms.com

**Author for correspondence: hjan@cnu.ac.kr

newlands press part of 
strategies, techniques and instrumentation, currently used for evaluation of biopharmaceuticals and immunogenicity.

\section{Ligand-binding assay strategies: singlicate analysis \& tiered method validation}

Ligand-binding assays (LBA) are the most commonly used methods to measure protein concentrations and assess immunogenicity in biological samples. Only LBA was discussed in the 2007 European Medicines Agency (EMA) Guideline on the Clinical Investigation of The Pharmacokinetics of Therapeutic Proteins as an assay platform for bioanalysis of therapeutic proteins [13]. Over $95 \%$ of data assessing clinical pharmacokinetics (PK) of therapeutic proteins published up until 2013 had been obtained with LBA techniques [14]. Advances in LBA technologies and automation have not only significantly enhanced sensitivity, increased throughput and enabled analysis of samples collected via microsampling, but also generated data with less variability. In regulatory bioanalysis, an individual sample is typically assayed in replicates using LBAs, whereas singlicate analysis has long since become the accepted standard assay strategy using LC-MS and in the clinical diagnostic setting using autoanalyzer platforms. While singlicate analysis in LBAs for the quantification of protein drugs and antidrug antibodies (ADAs) in a regulated environment has been discussed for quite some time, wider acceptance of this strategy is still lacking. The Editorial article by Birnboeck et al. points out that LBA technical platforms have reached a mature status in terms of assay variability and identifies the areas in which the singlicate analysis approach can best provide benefits without loss of data quality [15].

Bioanalytical methods must enable the timely delivery of data that adequately meet scientifically justified and fit-for-purpose criteria. The bioanalytical community tends to validate PK assays that surpass what is scientifically critical at discovery or early development stage of biotherapeutic development, thus, taking an unnecessarily length of time and costing more to develop and validate a new PK assay. Watson et al. proposed three tiers of method validation (regulatory, scientific and research) for large molecule LBA methods in PK assessment, providing risk balanced approaches to ensure that fit-for-purpose methods support pharmacokinetic testing through all phases of biotherapeutic development [16].

\section{Biosimilars: ADA assay comparability testing}

Since the first biosimilar $\left(\right.$ Omnitrope $\left.{ }^{\circledR}\right)$ was approved by the EMA in 2006, more than 20 biosimilars have been approved by either the EMA or the US FDA [11]. Distinct from the small-molecule generics, they are complex large molecules and therefore, have brought new challenges especially to bioanalytical assays used to determine ADA. One example of the challenges is how to demonstrate that the ADA assay can adequately detect antibodies against both biosimilar and originator. There are still gaps in current guidelines with no full consensus in the industry or literatures about the choice between a one- and a two-assay approach, assay requirements and nature of positive controls. Ryding et al. recommend a one-assay approach that requires a minimum of two separate experiments, named as 'titration compatibility' and 'inhibition compatibility', for ADA assay comparability testing [17].

\section{Application of LC-MS technologies}

While LBAs are traditionally considered as a gold standard for protein bioanalysis, they are not sufficient to address all complex bioanalytical issues, especially when quality assay reagents are not available. Over the last decade, liquid chromatography coupled with mass spectrometry (LC-MS), has emerged as an appealing technology for therapeutic protein bioanalysis, complementing traditional LBAs $[18,19]$. Nevertheless, there are also significant challenges in isolating, concentrating, detecting and quantifying specific proteins by LC-MS in the presence of the overwhelming abundance of endogenous proteins found in biological matrices. As biotherapeutic protein analysis using LCMS technologies is an emerging area, this special focus issue includes four articles to present the advances and considerations of application of LC-MS for characterization and quantification of therapeutic proteins. The editorial article by Zhou et al. focuses on the parameters to improve sensitivity, outlining the factors affecting LC-MS detectability in protein bioanalysis and discussing how these can be optimized during the steps of selection of signature peptides, recovery of sample preparation and optimization of LC-MS [20]. Fu et al. describes considerations of use of LC-MS in tissue analysis [21]. In addition, two Research Articles detail approaches of use of LC-MS to characterize glycans of therapeutic proteins $[22,23]$.

\section{PTMs, glycan-induced ADA \& glycan analysis} The majority of proteins derived from eukaryotes undergo PTMs by changing either the chemical nature of an amino acid (e.g., glycosylation, deamidation, deimination and oxidation) or protein structures (e.g., S-S formation, and proteolytic cleavage). While proper PTMs are necessary for biotherapeutic production and function, undesirable PTMs can potentially cause issues leading to inconsistent drug efficacy and unwanted toxicity. In addition, PTMs (especially nonhuman-derived PTMs generated in a 
nonhuman cell background) may elicit or enhance immunogenicity in dosed subjects. Montjovent $e t$ al. describes the development and validation of a method to detect the presence of antibodies of a recombinant therapeutic protein containing nonhuman sialic acid Neu5Gc [24]. Specifically, the approach is designed to distinguish immune responses between reactivity toward Neu5Gc or the remainder of the protein by a competing step using either the protein without Neu5Gc or an irrelevant protein with the glycan epitope.

PTMs can occur not only in the expression systems, but also during purification, formulation and storage processes as well as in vivo after administration. After administration into animals or human subjects, therapeutic proteins may undergo additional PTMs, also referred as biotransformation, resulting in further structural modifications which may potentially impact on PK and pharmacodynamics. PTMs are unique and complex aspects pertinent to biopharmaceutics, and the technologies and strategies to evaluate PTMs and their effects in vivo are still in their infancy. Importantly, approximately $60 \%$ of biotherapeutics are glycosylated protein [3]. As a main cause of heterogeneity among therapeutic proteins, glycosylation was reported to affect pharmacological activity and immunogenicity [25-28]. Current glycosylation profiling on biotherapeutics has mainly been focused on $\mathrm{N}$-glycosylation [28]. On the contrary, $\mathrm{O}$-glycan analysis is still challengeable due to the lack of commercially available specific enzyme for releasing $O$-glycans, potentially destructive effect of chemical releasing methods and interference of $N$-glycans. Kim et al. and Seo et al. described novel analytical methods for profiling of $O$-Glycans on recombinant human erythropoietin and recombinant coagulation factor IX (rFIX), respectively [22,23]. Although the work is not in the traditionally defined scope of bioanalysis, the technologies and methodologies can be potentially adapted to the evaluation of glycan-related biotransformation and glycan biomarkers in biological samples to understand the effects of glycosylation on therapeutic protein exposure, biomarker function and immunogenicity. In addition, it is important for bioanalytical scientists to understand the characteristic properties and how the critical quality attributes have been monitored for assay reference standards, reagents and analytes.

\section{Tissue analysis}

The need of an in-depth understanding of biopharmacuetical pharmacology including drug exposure at the target site of action, drug binding to the target and functional modulation at intended tissues has cata- lyzed the development of techniques of analyzing protein and other biotherapeutics in solid tissue samples. In addition, the expression levels of the target receptors and other receptors (e.g., FcRn) in the tissues can impact drug disposition and facilitate PK/PD modeling. Bioanalytical approaches for measuring biotherapeutics and protein biomarkers in circulation were typically adapted to tissue bioanalysis. However, these methods were not always transferable for tissue analysis to provide data with high confidence. Major challenges such as tissue heterogeneity, blood contamination and lack of controls for monitoring extraction efficiency remain to be addressed. Fu et al. [21] discussed some key considerations for LC-MS analysis of biotherapeutical and endogenous proteins in tissues and tips to address blood contamination, enhance extraction efficiency, improve sensitivity, and ensure quantitative accuracy and precision.

\section{Gene \& nucleic acid based therapeutics}

In addition to protein therapeutics, an increasing number of gene and nucleic acid based products have been advanced in clinical development phases $[7,8]$. While gene therapy is a promising treatment option for a number of diseases, the technique remains novel and the bioanalytical strategies remain to be defined. The review by Ma et al. discusses the recent practices and strategies in bioanalytical support of $\mathrm{PK} / \mathrm{PD}$ and immunogenicity evaluations in preclinical and clinical fields in gene therapy development with insight into method design, development and validations [29].

\section{Future perspective}

Advances in technologies in both LBA- and MS-based analytical platforms have not only made substantial contributions to quantifying and characterizing biotherapeutics and evaluating their immunogenicity in biologic fluids and tissues, but also enabled the bioanalytical community to adopt more efficient and higher throughput approaches to address complex bioanalytical issues in speed and without loss of quality. On the other hand, bioanalytical challenges still remain to be addressed due to the great complexity of biopharmaceuticals and biological matrices including heterogeneity associated with PTMs, aggregation, biotransformation and interaction with targets (e.g., free and bound), interference from sample matrices, ADAs, or co-administered drugs, and tissue sample heterogeneity. It is important to understand during assay development what forms of the biotherapeutics are most relevant to pharmacological assessment, which technology to use to measure the intended forms or variants, and to incorporate sound assay reagent gen- 
eration and characterization strategies. Furthermore, protein biologics almost exclusively target extracellular proteins due to limitations in intracellular delivery of large biomacromolecules [5]. This leaves many promising untapped intracellular targets, propelling significant efforts to develop proteins capable of accessing and binding targets inside the cell. Thus, innovative drug delivery strategies and novel complex modalities are expected to continue emerging, driving continuous bioanalytical technology innovation and strategy evolution. While in partnership with technology manufacturers to push on developing more sensitive, specific, efficient and automated technologies and approaches, it is critical that the bioanalytical community and regulators work in align-

\section{References}

1 Walsh G. Biopharmaceutical benchmarks 2014. Nat. Biotechnol. 32(10), 992-1000 (2014).

2 Chapman AP. PEGylated antibodies and antibody fragments for improved therapy: a review. Adv. Drug Deliv. Rev. 54(4), 531-545 (2002).

3 Bandaranayake AD, Almo SC. Recent advances in mammalian protein production. FEBS Lett. 588(2), 253-260 (2014).

4 Elvin JG, Couston RG, van der Walle CF. Therapeutic antibodies: market considerations, disease targets and bioprocessing. Int. J. Pharm. 440(1), 83-98 (2013).

5 Kintzing JR, Filsinger Interrante MV, Cochran JR. Emerging strategies for developing next-generation protein therapeutics for cancer treatment. Trends Pharmacol. Sci. 37(12), 993-1008 (2016).

6 Erickson BE. Untangling biosimilars. Chem. Eng. News 88(48), 25-27 (2010).

7 Stull RA, Szoka FC. Antigene, ribozyme and aptamer nucleic acid drugs: progress and prospects. Pharm. Res. 12(4), 465-483 (1995)

8 Patil SD, Burgess DJ. DNA-based biopharmaceuticals: therapeutics for the 21st Century. AAPS Newsmagazine 6(12), 27 (2003).

9 Cornes P. The economic pressures for biosimilar drug use in cancer medicine. Target. Oncol. 7(1), 57-67 (2012).

10 Declerck PJ, Simoens S. A European perspective on the market accessibility of biosimilars. Biosimilars 2012(1), 33-40 (2012).

11 Strand V, Girolomoni G, Schiestl M, Mayer RE, FricciusQuecke H, McCamish M. The totality-of-the-evidence approach to the development and assessment of GP2015, a proposed etanercept biosimilar. Curr. Med. Res. Opin. 33(6), 993-1003 (2017).

12 Schellekens H, Smolen JS, Dicato M, Rifkin RM. Safety and efficacy of biosimilars in oncology. Lancet Oncol. 17, e502-e509 (2016)

13 EMEA: Guideline on the clinical investigation of the pharmacokinetics of therapeutic proteins. European ment to evolve, harmonize and implement regulatory guidance and best practices to ensure scientifically sound, fit-for-purpose and efficient bioanalysis of biopharmaceutics.

\section{Financial \& competing interests disclosure}

The authors have no relevant affiliations or financial involvement with any organization or entity with a financial interest in or financial conflict with the subject matter or materials discussed in the manuscript. This includes employment, consultancies, honoraria, stock ownership or options, expert testimony, grants or patents received or pending, or royalties.

No writing assistance was utilized in the production of this manuscript.

Medicines Agency (2007).

http://www.ema.europa.eu/docs/en_GB/document_library/

14 Ezan E. Pharmacokinetic studies of protein drugs: past, present and future. Adv. Drug Deliv. Rev. 65(8), 1065-1073 (2013).

15 Birnboeck HF, Schick E, Justies N. Singlicate analysis in regulated bioanalysis using ligand binding assays: where are we heading? Bioanalysis 9(18), 1357-1359 (2017).

16 Watson RG, Egan AC, Schantz A et al. Implementing a tiered approach to bioanalytical method validation for large molecule LBA methods in PK assessments. Bioanalysis 9(18), 1407-1422 (2017).

17 Ryding J, Stahl M, Ullmann M. Demonstrating biosimilar and originator ADA binding comparability in ADA assays a practical approach. Bioanalysis 9(18), 1395-1406 (2017).

18 Papadimitriou A, Bansal S, Heinrich J, Staack RF. Can LC-MS/MS and ligand-binding assays live in harmony for large-molecule bioanalysis? Bioanalysis 6(13), 1735-1737 (2014).

$19 \mathrm{He} \mathrm{J}, \mathrm{Su} \mathrm{D}, \mathrm{Ng} \mathrm{C}$ et al. High-resolution accurate-mass mass spectrometry enables in-depth characterization of in vivo biotransformations for intact antibody-drug conjugates. Anal. Chem. 2017 (In press).

20 Zhou S, Vazvaei F, Ferrari L, Qu J. Practical considerations in enhancing LC-MS sensitivity for therapeutic protein bioanalysis. Bioanalysis 9(18), 1353-1356 (2017).

21 Fu W, An B, Wang X, Qu J. Key considerations for LC-MS analysis of protein biotherapeutics in tissues. Bioanalysis 9(18), 1349-1352 (2017).

22 Kim U, Oh MJ, Seo Y et al. Sensitive and comprehensive analysis of $O$-glycosylation in biotherapeutics: a case study of NESP. Bioanalysis 9(18), 1373-1383 (2017).

23 Seo Y, Park GM, Oh MJ et al. Investigation of $O$ glycosylation heterogeneity of recombinant coagulation factor IX using LC-MS/MS. Bioanalysis 9(18), 1361-1372 (2017).

24 Montjovent M-O, Zou L, Faust H, Abdolzade-Bavil A, Liu PM, Struwe P. Method establishment for discerned immunogenicity assessment of a recombinant glycoprotein containing non-human sialic acid Neu5Gc residues. Bioanalysis 9(18), 1385-1393 (2017). 
Parr MK, Montacir O, Montacir H. Physicochemical characterization of biopharmaceuticals. J. Pharm. Biomed. Anal. 130, 366-389 (2016).

26 Berkowitz SA, Engen JR, Mazzeo JR, Jones GB. Analytical tools for characterizing biopharmaceuticals and the implications for biosimilars. Nat. Rev. Drug. Discov. 11(7), 527-540 (2012).

27 Fekete S, Veuthey JL, Guillarme D. New trends in reversedphase liquid chromatographic separations of therapeutic peptides and proteins: theory and applications. J. Pharm. Biomed. Anal. 69, 9-27 (2012).

28 Oh MJ, Hua S, Kim U et al. Analytical detection and characterization of biopharmaceutical glycosylation by MS. Bioanalysis, 8(7), 711-727 (2016).

29 Ma M, Balasubramanian N, Dodge R, Zhang Y. Challenges and opportunities in bioanalytical support for gene therapy medicinal product development. Bioanalysis 9(18), 1423-1430 (2017) 